Arts \& Humanities | Guangqing Chi

\section{\#SocialScience: Mining Twitter for Social and Behavioural Research}

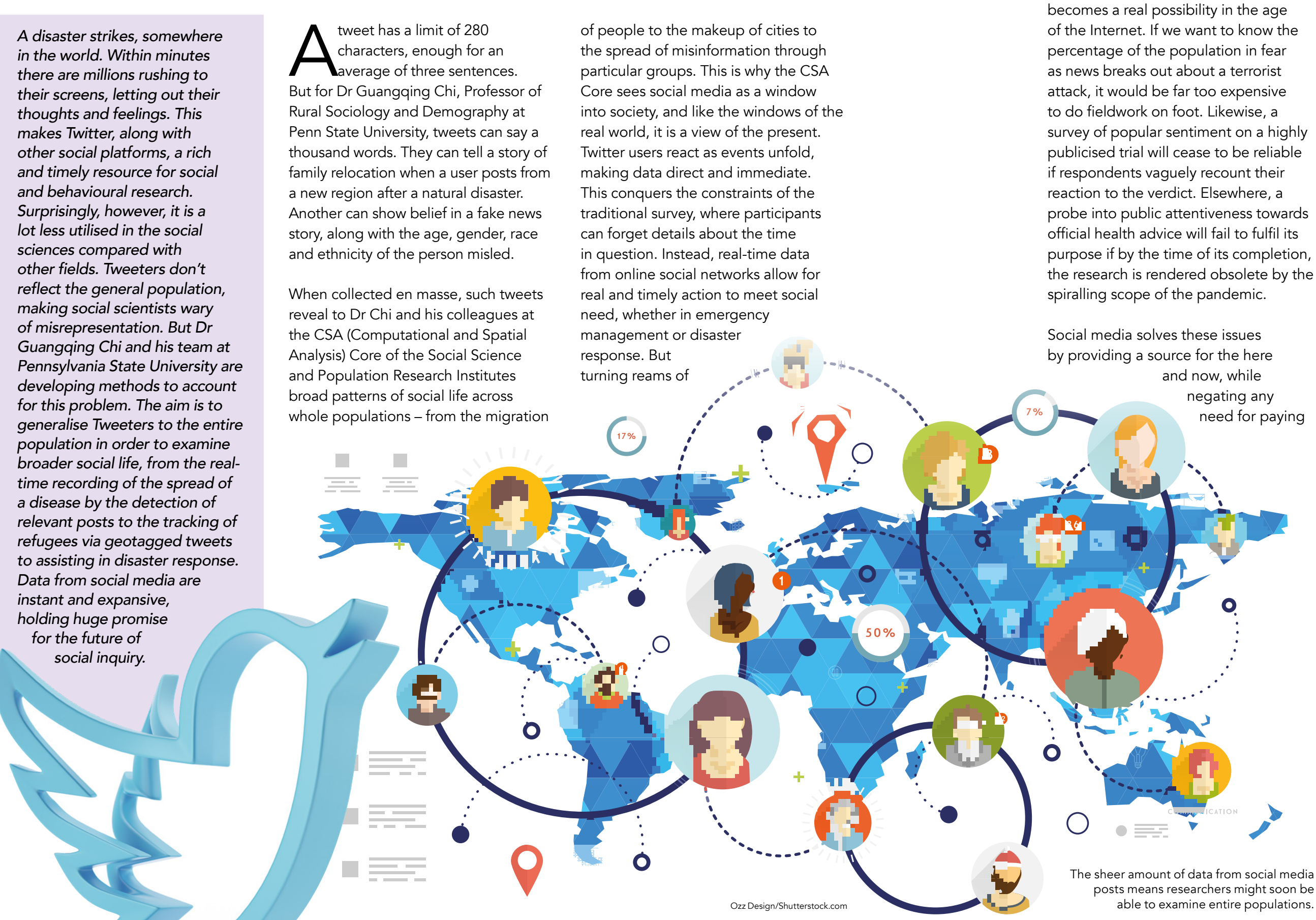

posts into a picture of wider societal phenomena requires sophisticated Core is at the forefront of building these tools, and the proiects they pursue are as engaging as they are effective.

At the click of a button, social media turns our lives into data for research. Minutes after an event erupts, people ace onto Twitter and other online platforms, to share with the world their motions and experiences. What was unfeasible with the conventional survey ecomes a real possibility in the age ying

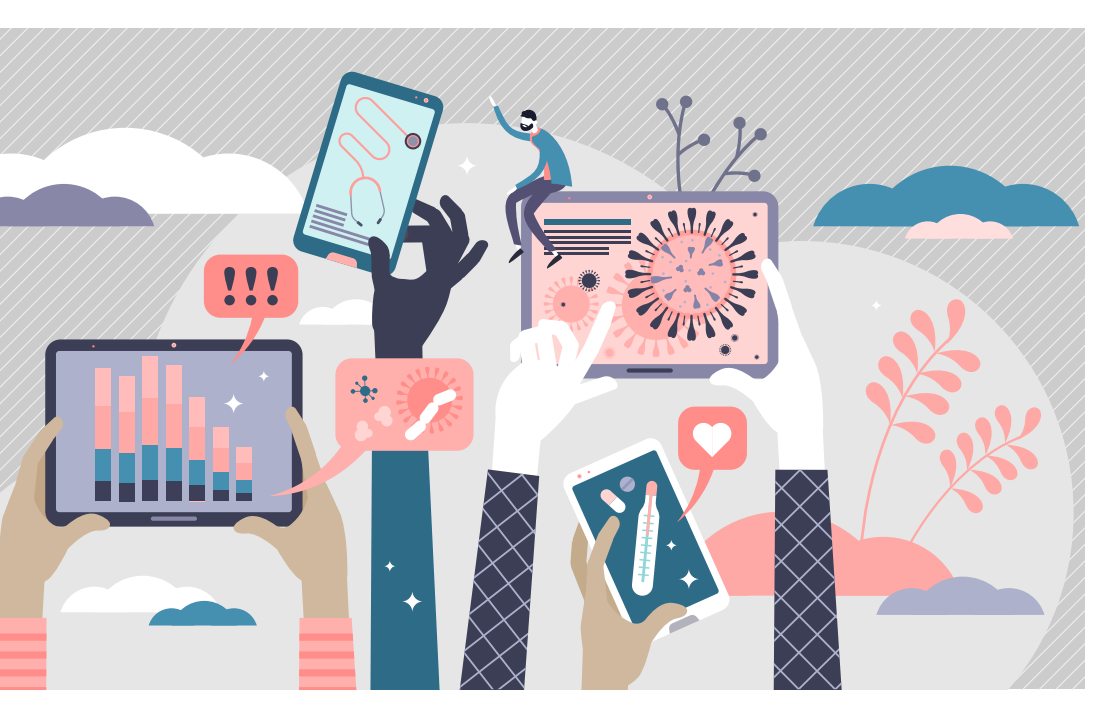

How do people react to official health advice regarding Covid

participants or funding legwo it doesn't stop there. Unlike 'data participants and plan questions, the Twitter posts and the Facebook profites are 'found in the wild'. The organic nature of online communities makes for spontaneous ties and tensions, whether in friendship, political debate, or criticism of the culture. This banishes the bias where subjects pander to the researcher, which can

Social media is more than the sum of its posts. Every user has a unique path

mount of data put into the hands of the social scientist. There are 2.5 billion sers of online social networks, and with he right methods to wrestle with this aterial, they can be used to this research is therefore vast in scope, so it's unsurprising to have drawn such eager attention - from the sphere of public health to political science. But to see clearly through this window into our society, an institution must first wipe the mist that obscures our view. The CSA Core at Penn State is committed to this task, refining statistical techniques and ways of weighting data, to reveal how

There are $\mathbf{2 . 5}$ billion users of online social networks, and with the right methods to wrestle with this massive resource, they can be used to examine entire populations.

through time in social research. The unfolding information can uncover how people are primed for certain content, or their movement across the world rom their changing tagged location. The turns the tracking of single users into a living document. Indeed, tomorrow's measurements of social media could display the dynamics of migration even before the release of official records. But the biggest and most basic value
MAKING WAVES IN METHODOLOGY

Scrolling through a top trend on Twitter does not reflect the general population. Users are skewed towards the young, the middle-class, and the male, which is far from representative of an entire fown or cily. Meanwhile, many members of social platforms are a demographic mystery, and without a way to unmask heri identiss, such data becomes usseless and is omitted from research. For 


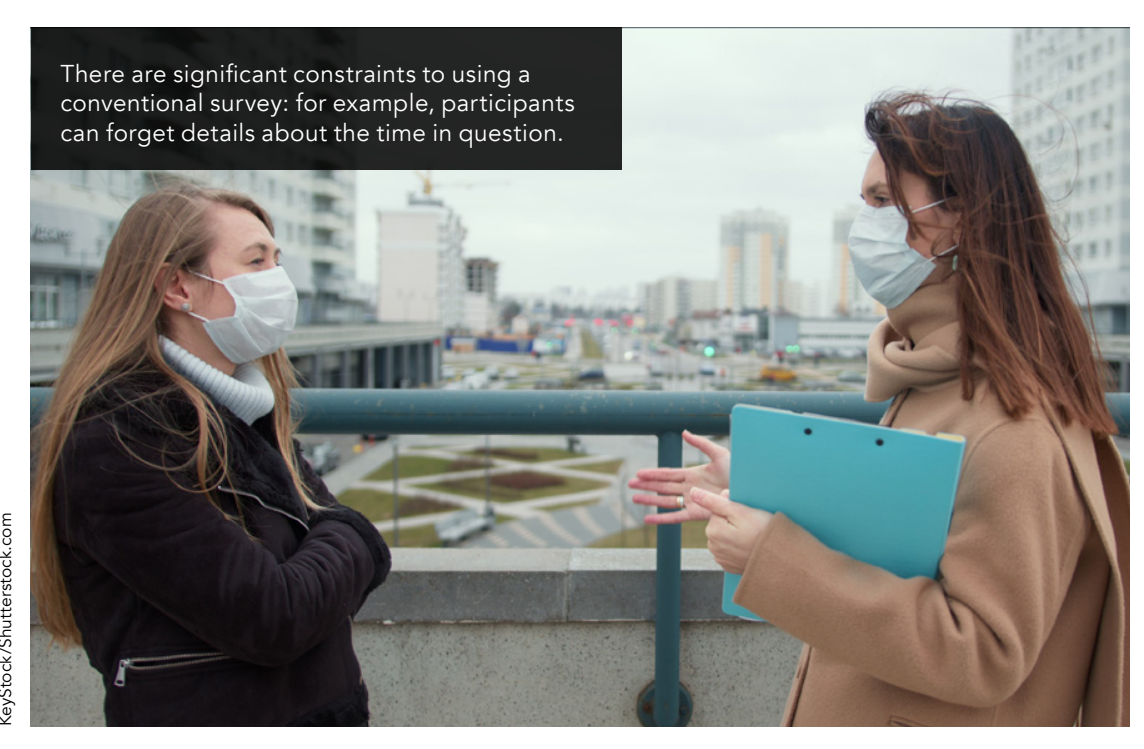

With real-time social media data, official messages could be swiftly improved through immediate intelligence on public reaction.

diminished confidence in social surveys as a means of drawing conclusions about the broader public. But for researchers at the CSA Core, these studies are not a lost cause. The group seeks to build and 'where' of Tweeters, improving techniques for assigning gender, age, race, and location. Analysts can then find bias that is buried in the data, where certain groups are under or overrepresented. Findings can be weighted and brought into the right balance, making Twitter samples representative terrain of Twitter resean is scatter with mines Researchers must deal wh the complexities of slang, sarcasm, and esoteric communication - from hashtag to emoticons to acronyms. Meanwhile, some Twitter users are not human but 'bots' - automated programs designed to advertise and misinform. Finding what is real and what is fake in these massive datasets adds another hurdle to an

already arduous challenge.

The research group uses multiple methods to identify Twitter users. One approach asks, "what's in a name?", as researchers detect gender and ethnic in usernames and screen names. Entries are compared against public name databases, from phonebooks to now invaluable for saving people's lives the cornavirus disele diagnosed with been widely recorded since the start of the outbreak. However effects on human behaviour and social dynamics have received little study so far. This is a glaring gap when such findings would allow us to track public awareness of the disease, the following of social distancing, attitudes toward lockdown and anti-Asian sentiment, among other important factors. Indeed, with real-time data from social media plattorms, officia messages could be swiftly improved through immediate intelligence geotagged tweets reporting symptoms d confirming cases have been used to predict new contractions of the size, up the five days in advance. The size of the sample is also so large that neighbourhoods, and the finest of time intervals, when scouring social networks for mentions of the disease. The Core is breaking new ground in this area with their online dashboard (https://webgis. pop.psu.edu/COVID-19), which can track these geotagged tweets across time and space.

The capacity of the CSA Core to gain Immense insight from tracking Twitter is clearly demonstrated by their range of research projects. The team is currently compiling geotagged tweets from a sixyear period to renew existing methods of detecting demographics. These techniques will be tested for reliability Puerto Rico after Hurricane Maria and comparing findings against records from the Internal Revenue Service of the U.S. Moreover, grants have bee obtained for other exciting studies - from an inquiry into inclination towards fake news to the food, energy, and water choices of Alaskans to the regional differences in opinion towards climate change. Social science stands at the foot of a mountain, where an expanse of evidence could be opened for analysis. The CSA Core is scaling the summit and seeking new opportunities to apply and extend their expertise. After all, if tweets from one user can say a thousand words, a million can make for research that eft

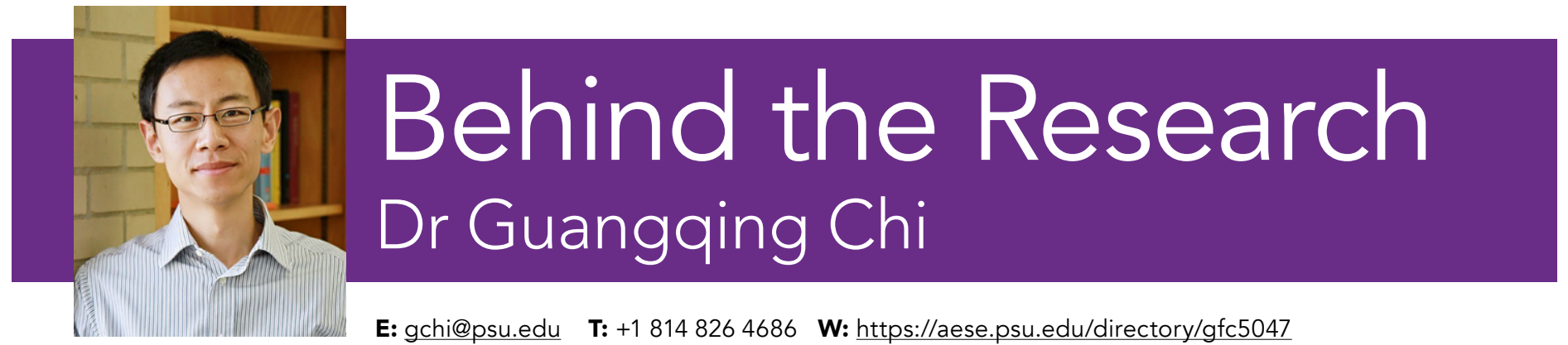

\section{Research Objectives}

Prof Chi gathers social media data in order to analyse real-time responses to social problems and phenomen

\section{Detail}

Guangqing Chi

112E Armsby

PA 16802

USA

Bio

Dr Guangqing Chi is Professor of Rural Sociology and Demography and Director of the Computational and Spatial Analysis Core at Pennsylvania State University. His research is focused on socio-environmental system aiming to understand the interactions between human populations and built and natural environments.

Funding

National Science Foundation (\#1823633 and \#1541136) and Social Science Research Institute, Population Research Institure, and Instiutre for Computational and Data Sciences, Pennsylvania State University

Collaborators

- Dr Junjun Yin, Computational and Spatial Analysis

Core, Pennsylvania State University

- Prof Jennifer Van Hook, Department of Sociology and

Criminology, Pennsylvania State University

- Prof Eric Plutzer, Department of Political Science,

- Prof Heng Xu, Kogod School of Business,

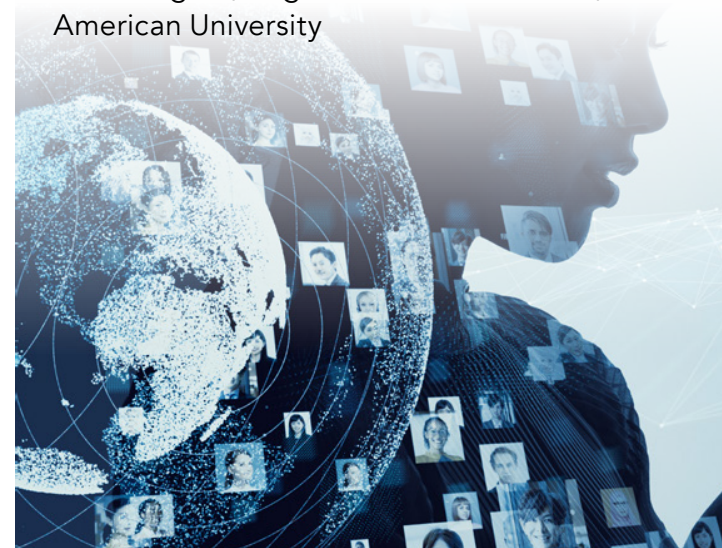

\section{References}

Auman-Bauer, K. (2020). Penn State researchers evaluating Twitter data during pandemic. [online] PennState. Available at: https://bit.ly/3go5wZA [Accessed 26 May 2020].

Auman-Bauer, K. (2019). Seed grants awarded to projects using Twitter data. [online] PennState. Available at:
https://bit.ly/2M3jySo [Accessed 26 May 2020].

Chi, G., Yin, J., Hook, J.V., Plutzer, E., Xu, H. (2019). The Generalizability of Twitter Data for Population Research. Population Association of America. Available at: https://bit.ly/2X0xROy [Accessed 26 May 2020].

\section{Personal Response}

Do you think your work, particularly the 'global dashboard, could be useful for public

II Twitter data can be greatly useful for public health research, if used correctly. By following individual potential to allow researchers to track public healthrelated phenomena such as the COVID-19 outbreak and public awareness, the effectiveness of policy interventions, and more. As the data can be streame in near real time, they provide timely information situation and make data-informed precision decisions. That said, the data need to be used in a rigorous and careful way. For example, the demographic characteristics of the Twitter users versus the targeted
population must be considered. 